\title{
Investigation of Wall Normal ElectroMagnetic Actuator for Seawater Flow Control
}

\author{
Lionel Rossi and Jean-Paul Thibault \\ LEGI $^{\dagger}$, BP 53, 38041 GRENOBLE, France \\ lionel.rossi@hmg.inpg.fr or lionel.rossi@netcourrier.com \\ jean-paul.thibault@hmg.inpg.fr \\ http://www.legi.hmg.inpg.fr
}

\begin{abstract}
ElectroMagnetic (EM) Flow Control deals with the concept of using in combination "wall-flush" electrodes (j, DC current supply) and "sub-surface" magnets ( $\mathbf{B}$, magnetic induction origin) to create directly local body forces $(\mathbf{j} \mathbf{x} \mathbf{B})$ within a seawater boundary layer. Analytical, experimental and computational investigations of EM Flow Control are presented here. This work is intended to understand the basic mechanisms involved in turbulence intensity and skin friction reductions as well as in coherent structure extinction. First, EM actuator and its modes of action are described. This description includes: some general remarks on EM actuator; the set of equations suitable to EM control in seawater; and a selection of dimensionless parameters analysed in term of possible mechanisms of action. Second, some experimental investigations and visualizations of wall bounded flows under EM actuation are presented: near wall vortex around the actuator; suction zone above the actuator; wall jets around the actuator; boundary layers "suction - blowing".
\end{abstract}

PACS numbers: 47.27.Rc, 47.65.+a

$\uparrow$ LEGI is a joint laboratory of the Centre National de la Recherche Scientifique (CNRS), Université Joseph Fourier and the Institut National Polytechnique de Grenoble.

\footnotetext{
${ }^{1}$ This article was chosen from Selected Proceedings of the Second International Symposium on Turbulence and Shear Flow Phenomena (KTH-Stockholm, 27-29 June 2001) ed E. Lindborg, A. Johansson, J. Eaton, J. Humphrey, N. Kasagi, M. Leschziner and M. Sommerfeld.
} 


\section{Contents}

\section{Introduction 2}

2 EM actuator and its modes of action 3

2.1 Generality 3

2.2 Equation suitable to EM control in seawater 4

2.3 Dimensionless parameters suitable to EM control in seawater 5

2.4 Typical distribution of forces above a wall-normal EM actuator 6

2.5 Typical shape of the flow developed above an EM actuator 8

3 Experimental investigation of wall normal actuators 9

3.1 Flow initially at rest: near wall vortex around the actuator 9

$\begin{array}{ll}3.2 \text { Flow initially at rest: suction zone above the actuator } & 10\end{array}$

$\begin{array}{ll}3.3 \text { Flow initially at rest: wall jets around the actuator } & 13\end{array}$

$\begin{array}{ll}\text { 3.4 Flow in a seawater tunnel: boundary layers visualization } & 14\end{array}$

4 Introduction $\quad 15$

\section{INTRODUCTION}

ElectroMagnetic (EM) Flow Control deals with the concept of using in combination "wall-flush" electrodes (j, current density) and "sub-surface" magnets (B, magnetic induction) to create directly local Lorentz body forces $(\mathbf{j} x \mathbf{B})$ within a seawater boundary layer. Close to the wall, these $\mathbf{j} \mathbf{x} \mathbf{B}$ forces can act directly on velocity and vorticity components.

The electromagnetic Forces distribution can be managed either for i) drag reduction or ii) local prevention of specific events like flow separation and/or structure production. Nosenchuck \& Brown 1993 [1] have shown significant turbulent intensity reduction and drag reduction using a network of wall normal EM actuator, (see Figure 1(b)). Henoch \& Stace 1995 [2] and Weier et al 2000 [3] have shown a flow separation prevention using electrodes and magnets both parallel to the mean flow direction, producing Lorentz forces parallel to the wall.

At least two different approaches are possible for flow control by the means of Lorentz forces. First, local schemes (closed loop control) are meant to detect and suppress a turbulent event by "injecting" body forces as soon as it passes over an actuator, see $[4,5,6,7]$. Second, global schemes (open loop control) are meant to break the self-sustaining of wall turbulence by imposing novel velocity and vorticity components in the wall region.

At the present stage, no deep understanding has been extracted from published contributions explaining how EM flow control works. The present contribution is aimed at providing a step-by-step comprehensive model of the physical mechanisms involved in electromagnetic flow control. Combined analytical and experimental approaches, are progressively upgraded to give an actual description of a very complex reality and finally to get a more predictive scheme. 


\section{EM ACTUATOR AND ITS MODES OF ACTION}

\subsection{Generality}

In this work a group of two permanent magnet poles and two electrodes are called EM actuator, see Figure 1(a)\&(d) both components are flush to the wall. Figure 1(a) schematics a typical wall normal EM actuator. The magnetic field is generated by the permanent magnets ( $\mathrm{N}$ and $\mathrm{S}$ ). The positive and negative electrodes are placed between and perpendicular to the magnets. The distances between respectively magnets and electrodes are quite the same thus the actuator is "square". Forces are wall normal above the centre of actuator and significant vorticity sources occurred directly above the magnets and electrodes due to curl(jxB) sources (see Figure 1(b)). More precisely the computed 3D EM forces distribution (see Figure 2) shows that forces are wall normal above the centre of actuator and are 3D centripetal all around. In addition, considering practical large scale applications, this type of EM actuator has to be multiplied in number instead of enlarged in size. The network presented on Figure 1(c) comprises several interconnected EM actuator. The electricity supply has, in the present case, an actuation cycle comprising 4 phases. At each phase, only a quarter (1/4) of the total number of actuators is active. Considering the time evolution of the cycle, from phase 1 to 3 or 2 to 4 and so on, the actuation appears as a wave like motion of EM forces pulses produced above each active actuator [1].
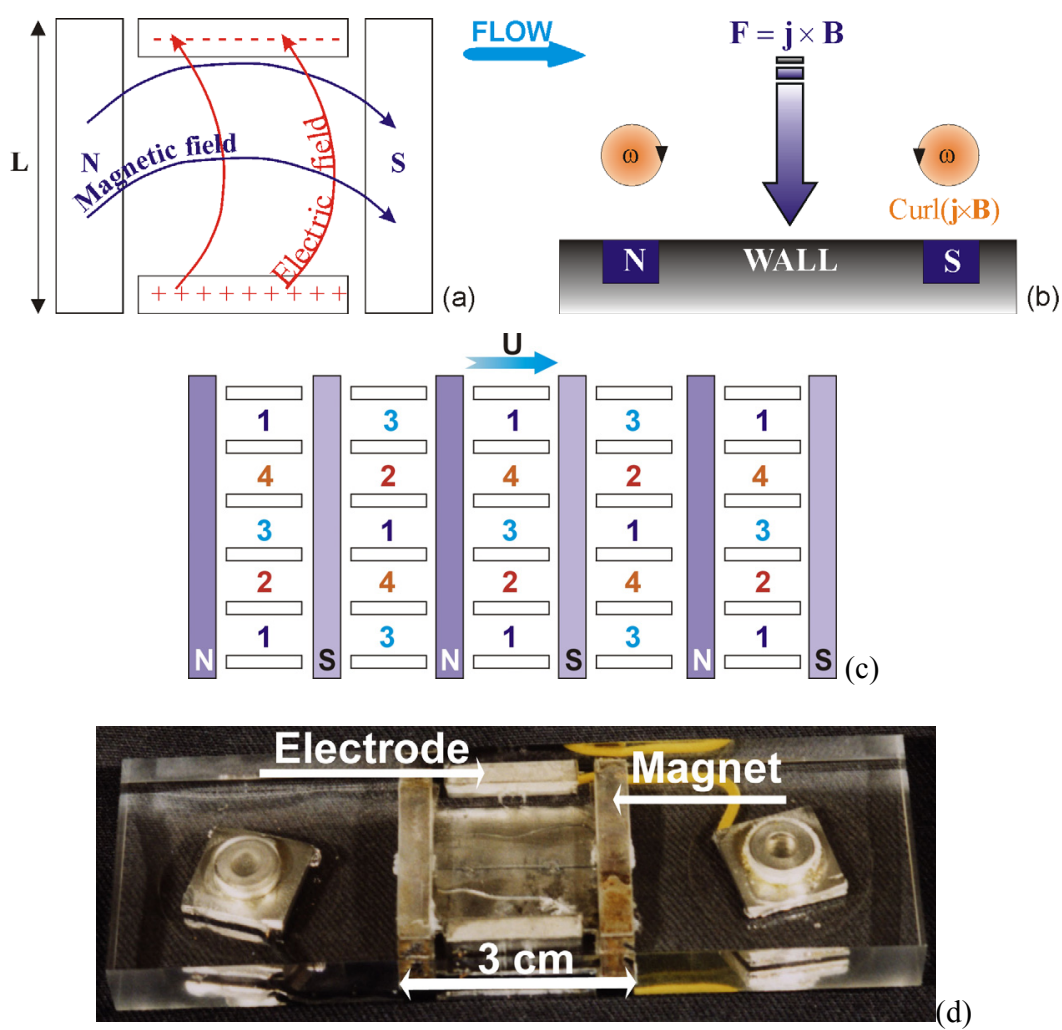

Figure 1: Wall normal EM actuator: a) Front view of magnets and electrodes arrangement at the wall. b) Cross section of magnets in wall. Sources of EM vorticity in the boundary layer due to Lorentz forces are indicated by $\omega$. c) Actuators network, top view illustration of the 4 first phases of electricity supply on a same board. d) Experimental EM Actuator 1999 


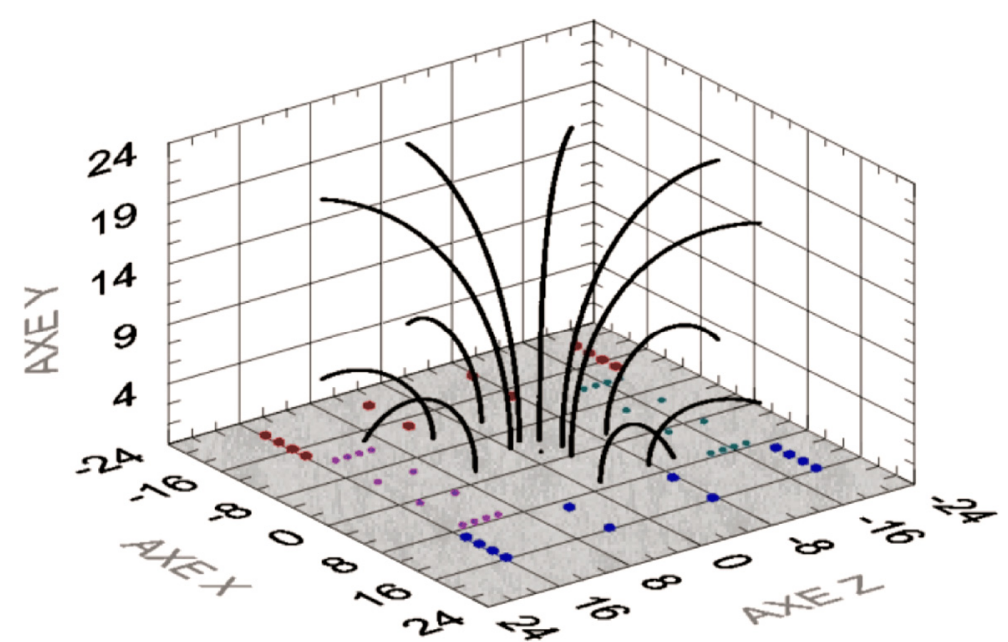

Figure 2: 3D view of computed EM forces lines above an actuator placed at the bottom plane of the plot.

To summarize, EM actuators can locally pump or act on the fluid in the wall region. This pumping is capable of producing novel velocity components as well as vorticity sources within the boundary layer. Contrary to "suction \& blowing" control [8] no mass flux through the wall is needed and the action is managed through the electricity supply of the electrodes.

\subsection{Equation suitable to EM control in seawater}

The following set of equations (see table 1) is aimed at describing properly the couplings between flow and electromagnetic fields. It is considered here that see water is a conductor having a conductivity $\sigma$ in its bulk (anywhere else than on the electrode surface) The governing fluid's equations are continuity (1) and Navier-Stokes equations (2) including the extra electromagnetic term due to Lorentz forces. The vorticity equation (3) is nothing more that the curl of (2). The existence of the right hand side term : $\operatorname{curl}(\mathbf{J} \times \mathbf{B})$ demonstrates that EM forces can act as a vorticity source. Equation (4) for magnetic induction, $\mathbf{B}$, in its final form reduces to a Laplace equation (4). This is due to first the use of permanent magnets and second to the very poor conductivity of seawater The latter giving a very low value to the magnetic Reynolds number (which measure the ratio of magnetic convection to magnetic diffusion). Ohm's law (5) is the constitutive equation for $\mathbf{j}$ the current density. It describes the balance between the electromotive field $\mathbf{u x} \mathbf{B}$ and the external electric field $\mathbf{E}$ derived from the electric potential (imposed at the electrodes). In the case considered the current density required has to be high enough to produce sufficient EM forces. It implies that, due to the moderate induction offered by permanent magnets, the imposed electric field is much larger than induced electric field. Consequently Ohm's law reduces to its simplified final form in the bulk flow of seawater $\mathbf{j}=\sigma \mathbf{E}$. Finally equations (6) express the conservation of both magnetic induction and electric current.

\begin{tabular}{|ll|l|}
\hline \multicolumn{1}{|c|}{ Fluid's equations } & \multicolumn{1}{|c|}{ Magnetic induction equation and Ohm's law } \\
\hline $\operatorname{div} \mathbf{u}=0$ & (1) & $\frac{\partial \mathbf{B}}{\partial t}=\mathbf{c u r l}(\mathbf{u} \times \mathbf{B})+\frac{1}{\mu \sigma} \nabla^{2} \mathbf{B} \Rightarrow \nabla^{2} B \approx 0$ \\
\hline$\rho \frac{d \mathbf{U}}{d t}+\nabla P+\rho \mathbf{g}=\mu \nabla^{2} \mathbf{U}+\mathbf{j} \times \mathbf{B}$ & (2) & $\mathbf{j}=\sigma(\mathbf{E}+\mathbf{u} \times \mathbf{B}) \Rightarrow \mathbf{j}=\sigma \mathbf{E}$ \\
\hline$\rho \frac{d \omega}{d t}=\rho \omega \cdot \nabla \mathbf{U}+\mu \nabla^{2} \omega+\nabla \times(\mathbf{j} \times \mathbf{B})$ & (3) & $\operatorname{div} \mathbf{B}=0$ and $\operatorname{div} \mathbf{j}=0$ \\
\hline
\end{tabular}

Table 1: Fluid, magnetic and electric equations suitable to seawater EM control 
It is remarkable that EM forces are sources terms which are capable of modifying the flow but are independent of the flow. They depend only on the actuator geometry and on the electric power supply.

\subsection{Dimensionless parameters suitable to EM control in seawater}

It is worthwhile to identify possible mechanisms of EM control in seawater and to this end, dimensional analysis is useful. The typical parameters chosen are: (i) Actuator length $\mathrm{L}_{\mathrm{EM}} \sim 10^{-2} \mathrm{~m}$, (ii) Magnetic induction $\mathrm{B} \sim 1 \mathrm{~T}$, (iii) Imposed electric field $\mathrm{E} \sim 10^{3} \mathrm{~V} / \mathrm{m}$, (iv) Electrical conductivity of seawater $\sigma \sim 5 \mathrm{~S} / \mathrm{m}$, (v) Seawater magnetic permeability $\mu=4 \pi 10^{-7} \mathrm{H} / \mathrm{m}$, (vi) Flow velocity $U \sim 1$ to $10 \mathrm{~m} / \mathrm{s}$, (vii) Boundary layer thickness $\delta \sim 10^{-3}$ to $10^{-2} \mathrm{~m}$. The Reynolds number $\mathrm{R}_{\mathrm{ex}}$ is of the order of $10^{6}$ or more. It is clear from the previous equations that EM parameters are independent of the flow (separate scales). Table 2 is constructed within the case of an action zone of EM forces larger than the boundary layer thickness, see $[9,10,11]$.

The first dimensionless parameter is the Hartmann number: Ha, equation (7). It measures the ratio of electromagnetic forces to viscous forces. In the present case Hartmann number is of order of one. It means that, in the boundary layer (thickness $\delta$ ), EM forces injected in the flow balances viscous terms. The second dimensionless parameters are interaction parameters I. They measure the ratio of EM forces to inertial forces. Concerning these parameters, it is particularly interesting to consider various scales or parameters length-scale and velocity-scale. The three cases presented here are respectively connected to external flow in equation (8), wall normal flow in equation (9), local velocity fluctuation in equation (10). It comes out from the typical values obtained here that the strongest interaction parameter is the one based the mean wall normal component (9). Thus the normal mean flow is expected to be dominated by the EM forces. On the contrary it is clear that the longitudinal external flow can not be significantly affected by EM forces (8). Finally the moderate value of the local interaction parameter (based on a local velocity fluctuation) (10) demonstrates that the considered EM actuator is not appropriate in size and power to directly compete with "a turbulent event". Let us say that such an actuator could be designed but it would be much smaller. In the meantime this micro-actuator has to be supplied where and when a turbulent event is detected, which is not the story of the present work.

\begin{tabular}{|c|cc|}
\hline \multicolumn{3}{|c|}{ Viscous parameter } \\
\hline$\frac{\text { EM term }}{\text { Viscous term }}$ & $H_{a}^{2}=\frac{\sigma E B \delta^{2}}{\rho v U} \approx 1$ & (7) \\
\hline \multicolumn{2}{|c|}{ Inertial parameters I : (Interaction parameters) } \\
\hline $\begin{array}{c}\text { Effect on } \\
\text { longitudinal } \\
\text { component }\end{array}$ & $I_{U}=\frac{\sigma E B}{\rho \frac{U^{2}}{\delta}}=\frac{\sigma E B \delta}{\rho U^{2}} \approx 10^{-4}$ to $10^{-2}$ & (8) \\
\hline $\begin{array}{c}\text { Effect on } \\
\text { normal } \\
\text { component }\end{array}$ & $I_{V}=\frac{\sigma E B}{\rho \frac{V^{2}}{\delta}}=\frac{\sigma E B \delta}{\rho V^{2}} \approx 10$ to $10^{4}$ \\
\hline $\begin{array}{c}\text { Effect on a } \\
\text { local velocity } \\
\text { fluctuation }\end{array}$ & $I_{v l o c}=\frac{\sigma E B}{\rho \frac{v^{2}}{l}} \approx 10^{-4}$ to $10^{-1}$ \\
\hline
\end{tabular}

Table 2: Non dimensional parameters associated with EM flow control 


\subsection{Typical distribution of forces above a wall-normal EM actuator}

After this brief description of general equations and dimensionless parameters, the following analysis of the geometry of the imposed EM forces is certainly very useful in order to get a better understanding of the possible modes of action of an EM actuator. The following results are based on numerical 3D computations of the EM forces field. These computations are based on an idealised description of electrodes as uniform sources of electric charges and of each magnet as two uniform sources of magnetic charges. This analytical solution, detailed in [11], is numerically computed at each point above the actuator.

$\partial$ The electromagnetic fields as well as the resulting forces developed above an EM actuator are 3D. A typical shape of forces lines $(\mathbf{f} x \mathbf{d l}=\mathbf{0})$ is shown in Figure 2. These lines have a centripetal distribution and are distributed like a "siphon shape". The direction of forces is mainly normal to the wall and their sign directly depends on current's sign (i.e. inward or downward the wall).

- The magnetic and electric fields both decrease from the wall $(y=0)$ towards the external flow. Therefore the magnitude of the forces is maximum at the wall. Numerical results on wall normal component of $\mathbf{j x} \mathbf{B}$ forces are showed on Figure 3. For a 1 Ampere current and a 1 Tesla magnetic induction, the wall-normal volume force: $\mathrm{f}_{\mathrm{y}}$ is of order of $-100 \mathrm{~N} / \mathrm{m}^{3}$. The Figure 3(a) represents a coloured forces distribution in a wall normal plane $(\mathrm{z}=0)$. One can observe that the spatial extension of the zone where EM forces are quite intense is as large as the actuator width, L (see Figure 1), and its thickness is about L/5. The Figure 3 (b) gives the transversal distributions of wallnormal force component for various y elevations. The Figure 3(c) gives the wall-normal distribution of the wall-normal force component on the axe of the actuator. It can be observed here that a substantial increase in the maximum force does exist between the experimental actuators used for the present work respectively: actuator 1999 \& acti2000. Both have almost the same size except higher and larger magnets for the second one giving a higher magnetic field.

$\div \mathbf{j} \times \mathbf{B}$ forces are rotational, they induce vorticity components which are distributed all around the EM actuator. This is easy to imagine when considering the very high gradient of forces marked above the magnet poles $(\mathrm{x}=+/-15 \mathrm{~mm})$ on Figure $3(\mathrm{~b})$. More convincing is the distribution of wall-normal EM vorticity sources given in Figure 4 which is a coloured cut view at $y=2 \mathrm{~mm}$ of this vorticity source $\omega_{\mathrm{EMy}}$. Finally Figure 5 (c) illustrates the volume, above the actuator, where the force's rotation is larger than 20 $\mathrm{rd} / \mathrm{s}^{2}$. 


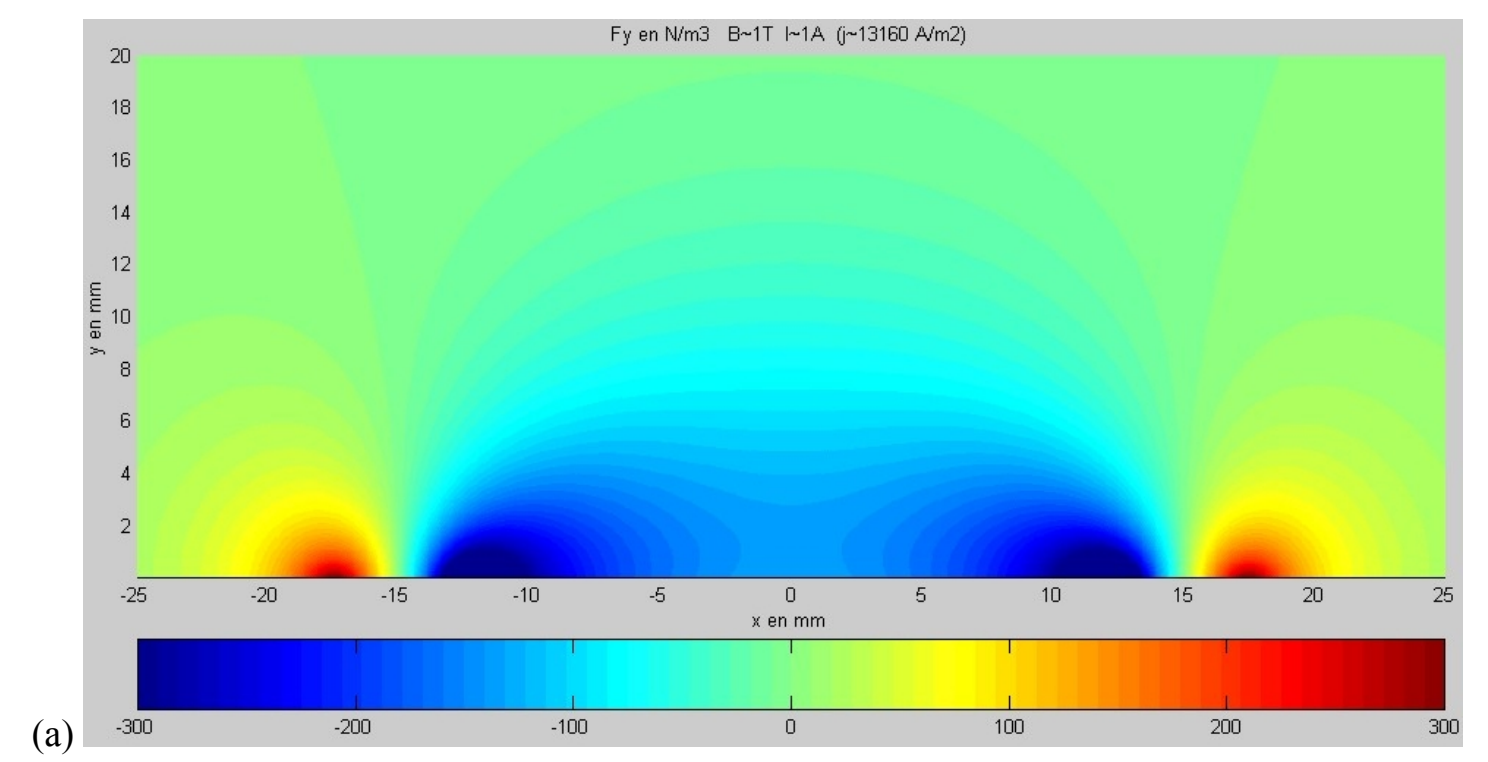

(b)
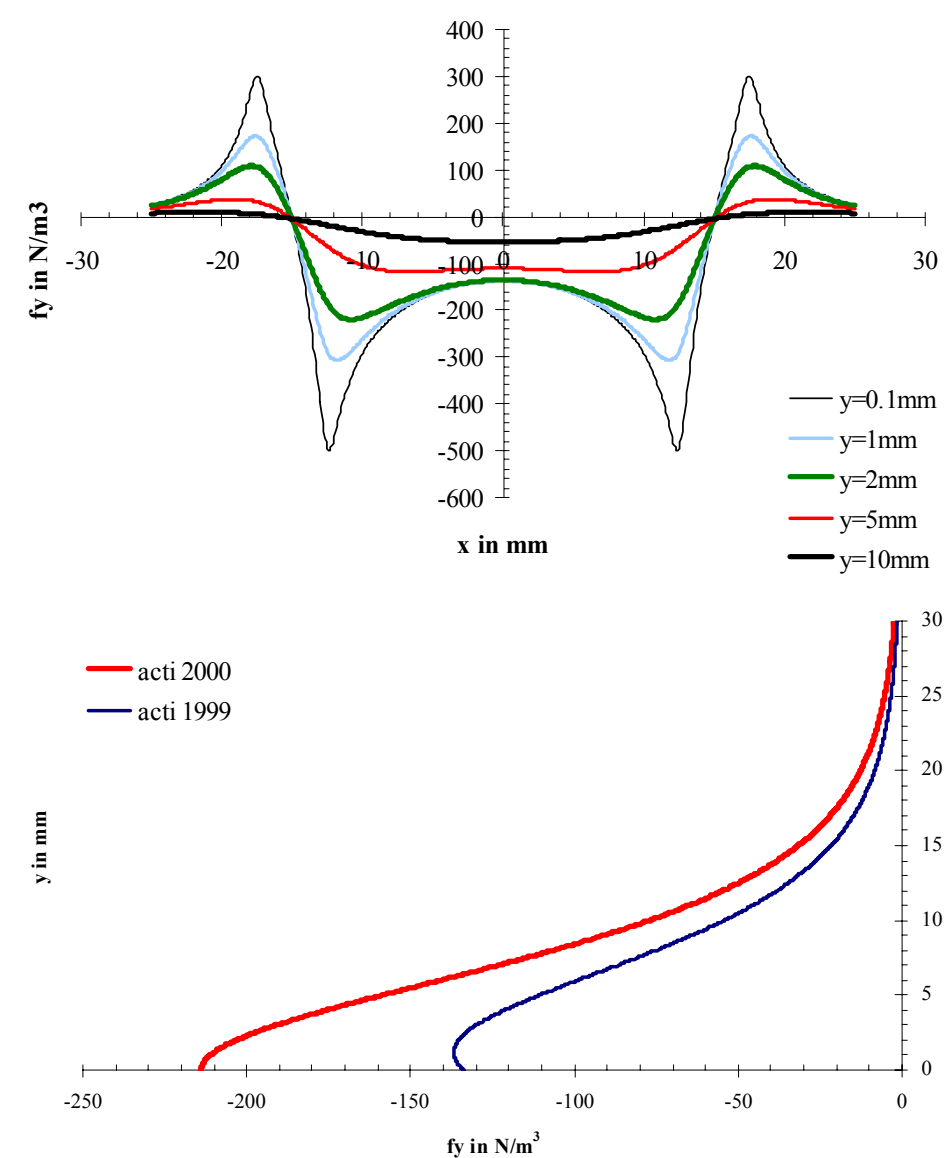

(c)

(axis: $\mathrm{x}=\mathrm{Z}=0$ )

Figure 3: Computed wall-normal component of EM forces $\left(\mathrm{Fy}\right.$ in $\left.\mathrm{N} / \mathrm{m}^{3}\right)$ in a plane normal to wall and magnet, at the centre line of EM actuator $(\mathrm{z}=0), \mathrm{B}=1 \mathrm{~T}, \mathrm{I}=1 \mathrm{~A}, \mathrm{~J}=13 \mathrm{160 \textrm {A }} / \mathrm{m}^{2}$ actuator 1999 : (a) Colourmap in the plane (x,y); (b) Transverse distribution at various y position; (c) Wall-normal profile on the actuator axis : comparison of actuator 1999 and 2000. 


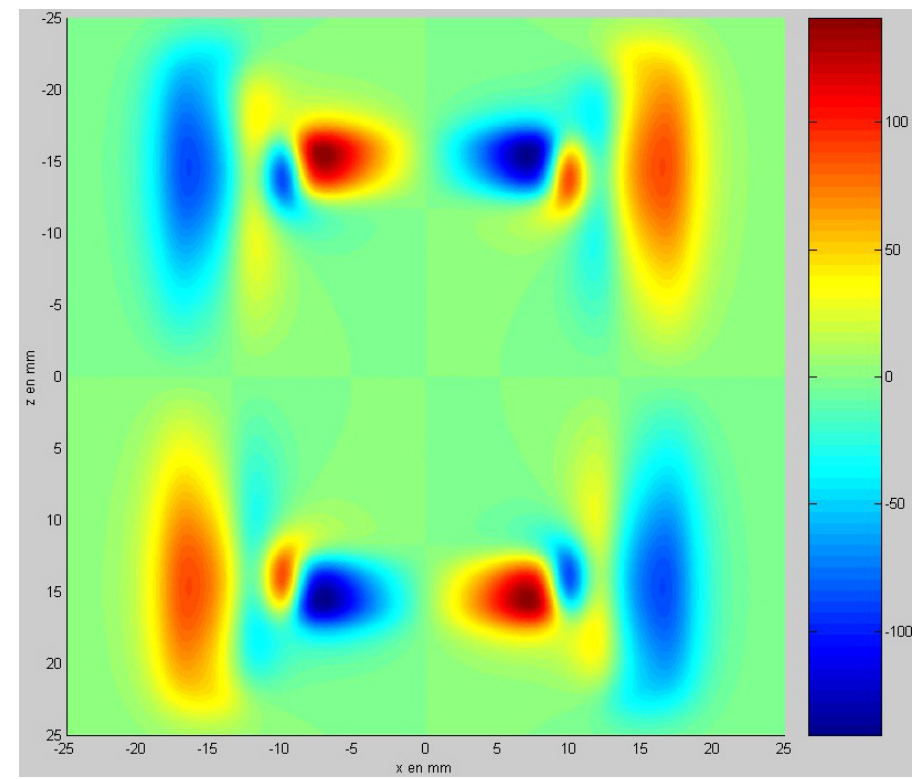

Figure 4: Colour-map of the computed EM vorticity source $\left(1 / \mathrm{s}^{2}\right)$ normal to the wall at $\mathrm{y}=2 \mathrm{~mm}$; $\mathrm{I}=1 \mathrm{~A}$, $\mathrm{B}=1 \mathrm{~T},-25<\mathrm{x}<25 \mathrm{~mm}$ and $-25<\mathrm{z}<25$. (Actuator 2000).

\subsection{Typical shape of the flow developed above an EM actuator}
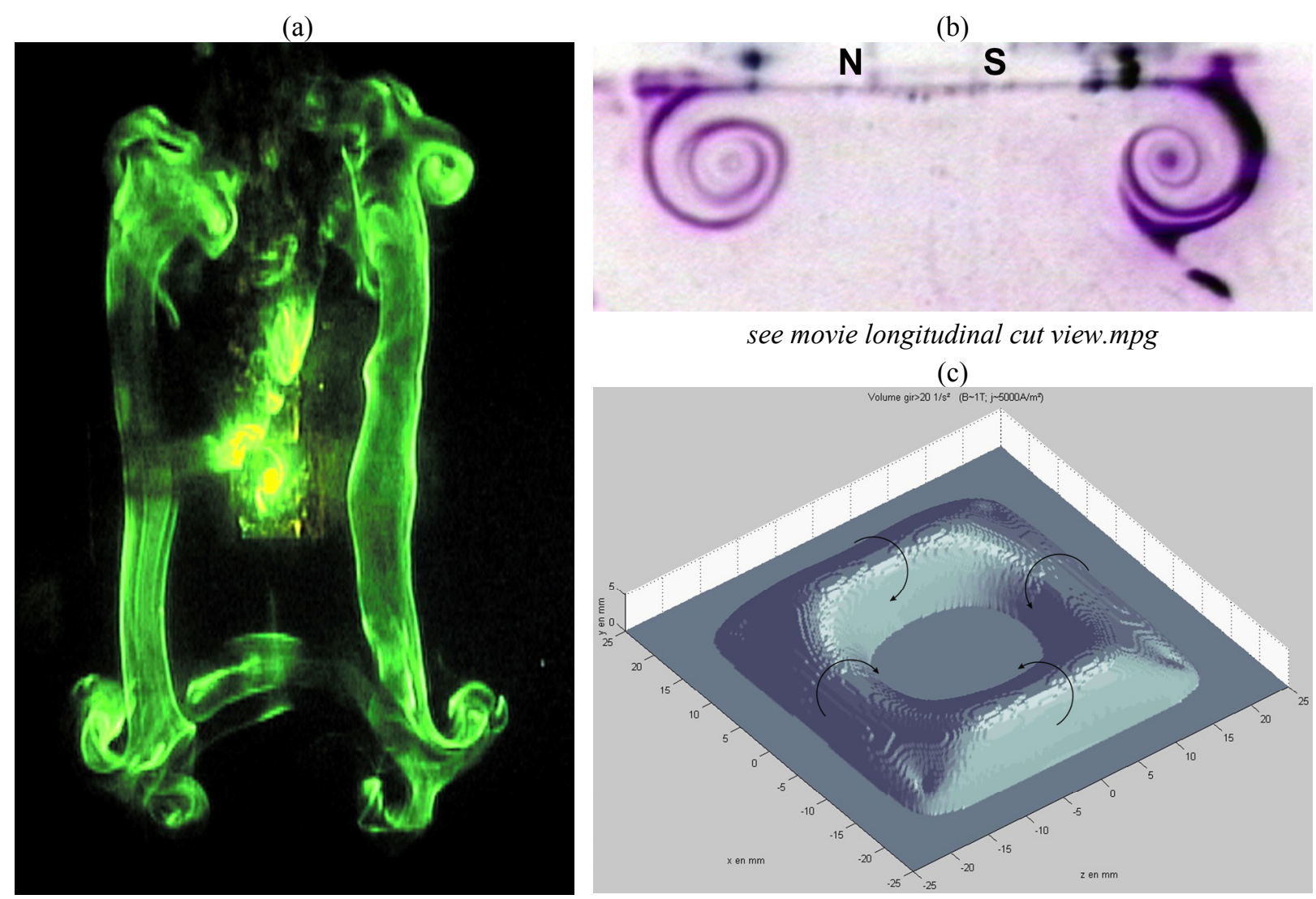

(c)

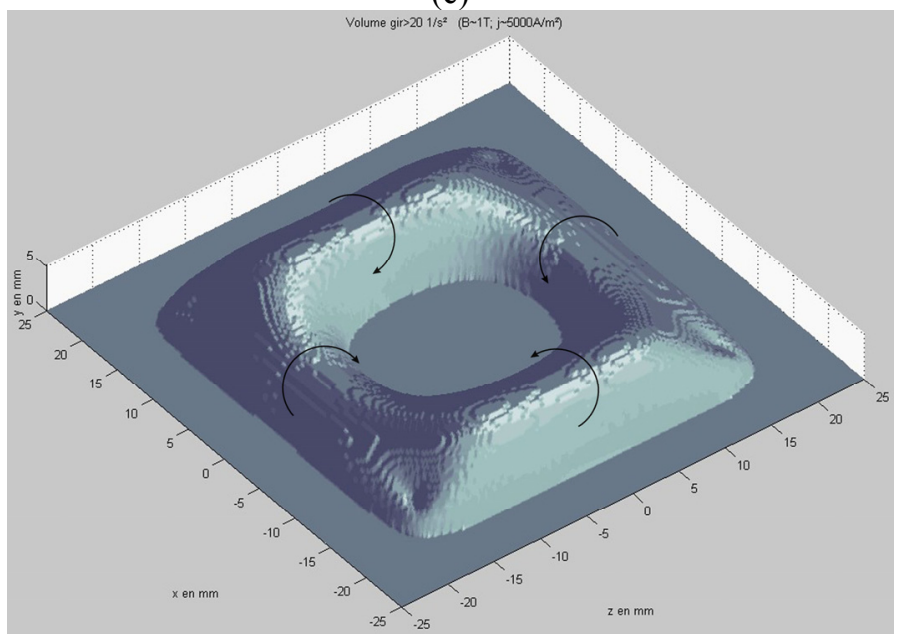

see movie normal view.mpg

Figure 5: (a)\&(b): Saltwater $(35 \mathrm{~g} \mathrm{NaCl} / 1)$ aquarium visualization: (a) Front view of vortical structure developing above the EM actuator in a flow initially at rest ( $\mathrm{I}=1.1 \mathrm{~A}$, time $\sim 5$ seconds, $\mathrm{B} \sim 0.65 \mathrm{~T}$ ); (b) Cut view of vortical structure developing above the EM actuator. (c) Computed 3D zone where EM forces rotation source is larger than $20 \mathrm{rad} / \mathrm{s}^{2}$ (given by $\|\mathbf{F}\| / \rho * \mathbf{C u r l}(\mathbf{F} /\|\mathbf{F}\|),[11]$ ); $\mathrm{B} \sim 1 \mathrm{~T}, \mathrm{I}=1.1 \mathrm{~A}$, $\mathrm{J}=14500 \mathrm{~A} / \mathrm{m}^{2}, \mathrm{~L}=30 \mathrm{~mm}$. (Actuator 1999) 
Figure 5 (a)\&(b) is the flow visualization (fluorescent die injection, see movies files: normal view.mpg and longitudinal view.mpg) of an experiment performed in an aquarium large enough to avoid confinement effects. The flow is driven by a continuous (5 seconds) EM actuation from a flow initially at rest. The top view (in a plane parallel to the wall) given in Figure 5(a) and the cut view (in a plane perpendicular to the wall) given in Figure 5(b), both demonstrate that the flow is formed of large coherent vortical structures. The typical length scale of these structures is ten times larger than the actuator length (L). Rotational tubes parallel to magnet or electrodes and mushroom shape in the angles can be noticed. These "mushroom shapes" can be explained by the normal EM vorticity source in the corners, see Figure 4 . In addition in the next part detailed measurements explain that these "mushroom shapes corners" are transported outward by intense wall jets located in the corners, (cf. Figure 11 and Figure 12).

\section{EXPERIMENTAL INVESTIGATION OF WALL NORMAL ACTUATORS}

The experiments reported are realized with "seawater" (35 g/l NaCl solution). A part of the experiments are performed in an aquarium $\left(50 * 60 * 50 \mathrm{~cm}^{3}\right)$ large enough to avoid confinement effects. The rest of the experiments are performed on a seawater test loop.. Two visualization test sections (tunnel) including a wall-flush EM actuator are used. The small tunnel $\left(4 \mathrm{~cm}^{*} 4 \mathrm{~cm}^{*} 100 \mathrm{~cm}\right)$ corresponds to a maximum velocity of $10.4 \mathrm{~m} / \mathrm{s}$ but confinement is very strong due to its reduced size compare to the actuator size $(3 \mathrm{~cm})$. The large tunnel $(10 \mathrm{~cm} * 10 \mathrm{~cm} * 130 \mathrm{~cm})$ corresponds to a maximum velocity of $1.66 \mathrm{~m} / \mathrm{s}$. In this case the confinement is sufficiently moderate for measurement purpose.

Two wall normal actuator are used. Both have wall-flush electrodes $(\mathrm{Pt} / \mathrm{Ti})$ with a $4 \mathrm{~mm} * 19 \mathrm{~mm}$ surface. Two different magnets are used: i) Actuator 1999 has a magnet $(\mathrm{NdFeB})$ of $8 \mathrm{~mm}$ height, $35 \mathrm{~mm}$ length and $5 \mathrm{~mm}$ width. ii) Actuator 2000 has a magnet $(\mathrm{NdFeB})$ of $20 \mathrm{~mm}$ height, $45 \mathrm{~mm}$ length and $5 \mathrm{~mm}$ wide. For each actuator the magnetic induction is about half the magnetisation of the material at the pole surface $(0.65 \mathrm{~T}=$ $(1.3 / 2) \mathrm{T}$ for $\mathrm{NdFeB})$ but due to the greater height of Actuator 2000 its useful magnetic field is typically $50 \%$ higher in the action zone see Figure 3(c).

The measurements presented hereafter are based on three experimental techniques: i) Flow visualization using fluorescent-die and light-plane, ii) Particle Tracking Velocimetry (PTV) iii) Particle Image Velocimetry (PIV); [10, 11]. The two first use conventional seeding and light-plane. The third one uses Rhodamine as seeding and planar laser (YAG) sheet. The optical filtering of Rhodamine fluorescence allows to eliminate the over-brilliancy of electrolysis gas bubbles.

\subsection{Flow initially at rest: near wall vortex around the actuator}

The measurements reported on Figure 6 are realized in the central wall normal plane $(\mathrm{z}=0)$ of the actuator just at the edge of the actuator $\mathrm{x}=13$ to $37 \mathrm{~mm}$. Two brief movies here attached (see movies files: velocity.mpg \& vorticity.mpg) give an animation of the PIV measurements of velocity and vorticity during the experiment. The flow is initially at rest in the transparent test section of the large tunnel $10 \mathrm{~cm}^{*} 10 \mathrm{~cm}^{*} 130 \mathrm{~cm}$. The vorticity is measured by PIV after a 3 seconds EM actuation. Figure 6(a) combines on the same plot vorticity scalar (color scale) and velocity's arrows. The measurements confirm visualization by fluoresceine (Figure $5(\mathrm{a}) \&(\mathrm{~b})$ ) and the presence of vorticity in the flow due to EM action. This vorticity field comprises the two constituents of vorticity: shear (or local rotation) and flow rotation. Near the wall, the wall jet imposes a shear type vorticity with alternative negative and positive signs and at some distance from the wall $(y=5.25 \mathrm{~mm}$ and $\mathrm{x}=30.86 \mathrm{~mm})$ a vortex core is clearly apparent. The 
triangular shapes of vorticity profiles (see Figure 6(b)\&(c)) clearly show that the observed structure is a complex vortex and not only a solid rotation. This PIV result complements visualizations of vortical structures observed during a 10 seconds DC actuation in an aquarium initially at rest, see $[9,10,11]$.

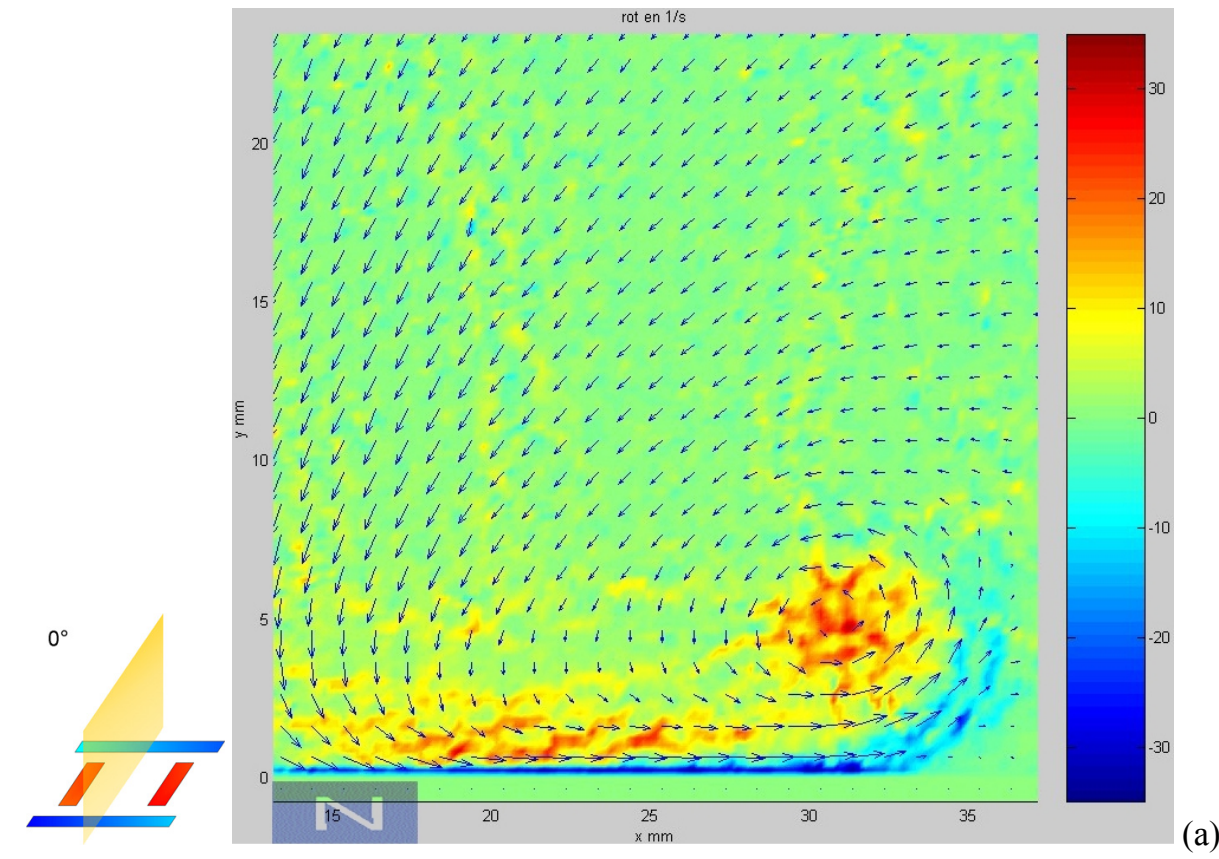

see movie vorticity.mpg \& velocity. $m p g$

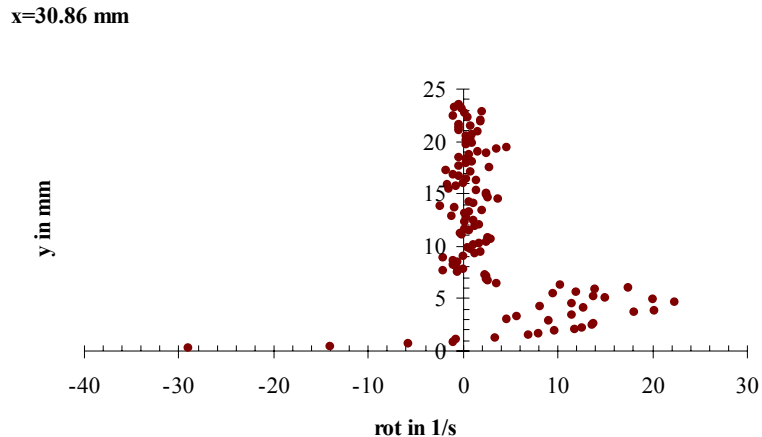

(b)

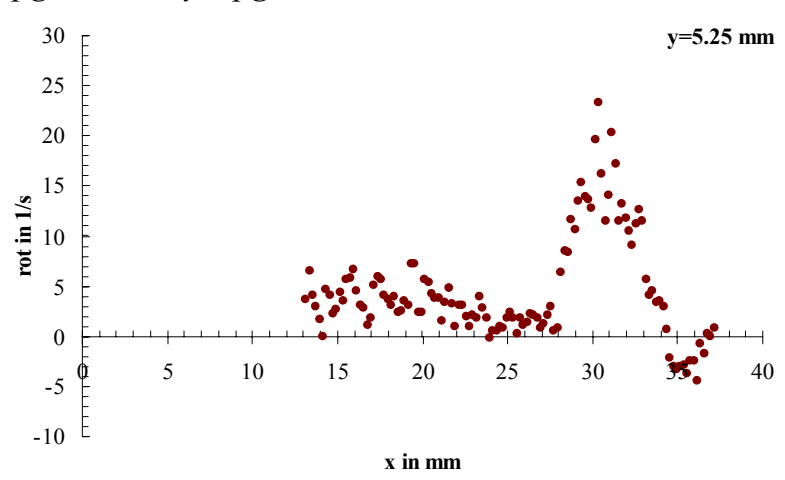

(c)

Figure 6: PIV measurement of vorticity after $3 \mathrm{~s}$ of actuation (flow initially at rest), I=1.73 A. Transparent test section of $10 \mathrm{~cm} * 10 \mathrm{~cm} * 130 \mathrm{~cm}$. (a): Colour-map of vorticity, arrows represent flow velocity, only one arrow for five measurements points is plotted. (b) \& (c): Profiles of vorticity in vertical and horizontal medians plane in the region of the vortex observable on the colour-map (Actuator 2000).

\subsection{Flow initially at rest: suction zone above the actuator}

The experiment is a 10 seconds actuation in an aquarium initially at rest. The brine is marked with particles that allow PTV measurements. After 10s of actuation the flow is quasi-developed. Figure 7 is the superposition of three frames delayed by about $0.2 \mathrm{~s}$.

PTV treatment of images, like in Figure 7, is presented on Figure 8(c). The measurements are realised in the central wall normal plane $(z=0)$ above the centre of the actuator. A large scale view of the flow $(8 \mathrm{~mm}<\mathrm{y}<50 \mathrm{~mm})$ shows that the so-called "suction zone", where EM forces pumped fluid, is much larger than attraction zone of EM forces, see Figure (a)\&(b). This is mainly due to flow continuity. 


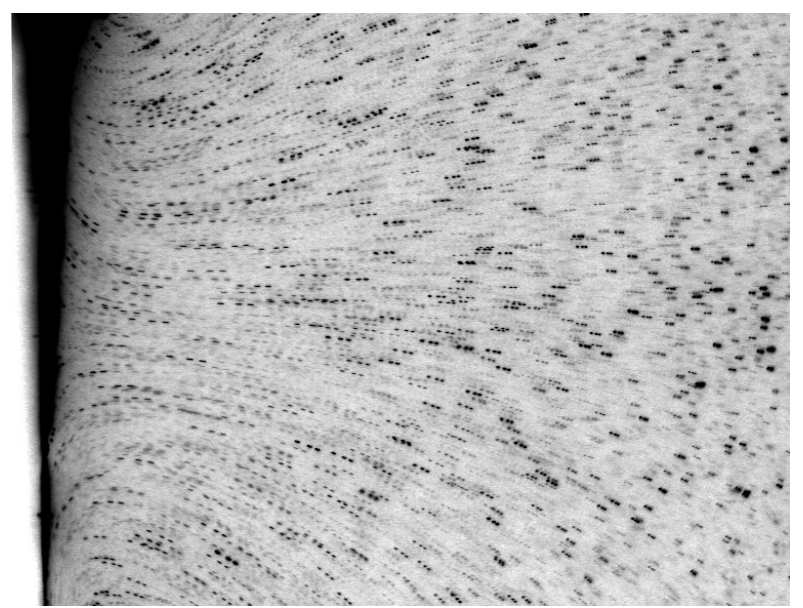

Figure 7: Particles visualization above the actuator in a vertical light sheet, 3 frames superposition, for $\mathrm{I}=1.1 \mathrm{~A} ; 10 \mathrm{~s}$ EM actuation (flow initially at rest), actuator 1999.

The Figure 8(c) superposes to the measured velocity field (marked by colour-scale and arrows) computed EM forces (marked by solid lines). The maximum suction velocity is about $14 \mathrm{~mm} / \mathrm{s}$ and is essentially normal to the wall. The suction zone is equivalent to the actuator size L, as well for its width as for its height. This suction zone (see Figure 8(b)) is larger than the zone where EM forces are strongly present (see Figure 8(a)), which height is only L/5.
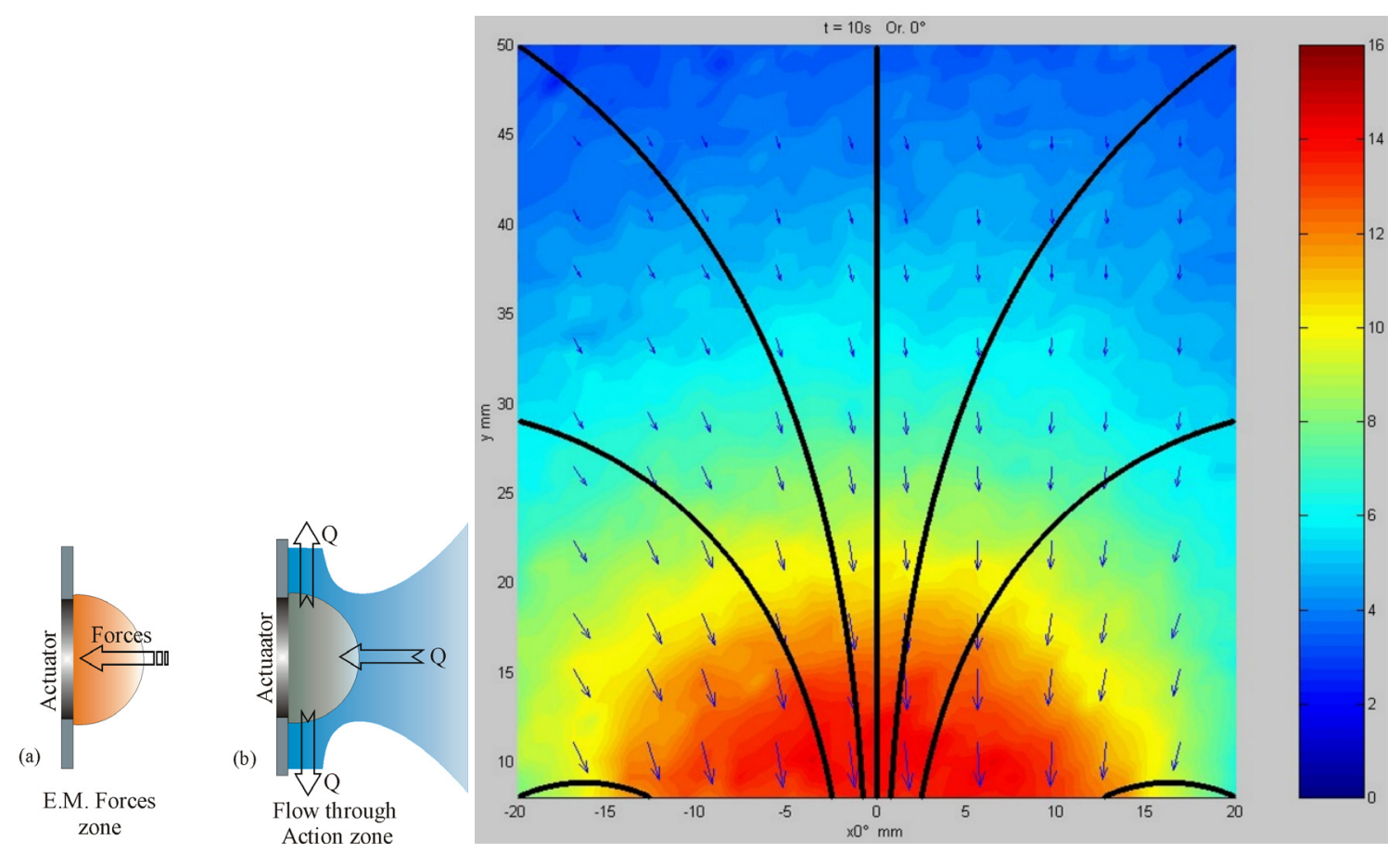

(c)

Figure 8: (a) \& (b): Schematic representation of action zones: (a) strong EM forces zone (b) EM pumped flow zone. (c) PTV of suction velocity (colour in $\mathrm{mm} / \mathrm{s}$ and arrows) and computed EM forces solid lines in a plane normal to wall and magnet, at the centre line of $\mathrm{EM}$ actuator $(\mathrm{z}=0)$; $\mathrm{B} \sim 0.65 \mathrm{~T}$ at magnets surface, $\mathrm{J}=14500 \mathrm{~A} / \mathrm{m}^{2}, \mathrm{I}=1.1 \mathrm{~A}, \mathrm{~L}=30 \mathrm{~mm}, 10 \mathrm{~s}$ actuation. (Actuator 1999)

The suction velocity increases as distance normal to the wall decreases. Of course this is due to increasing forces near the wall (see Figure 3(c)) but also to the integral effect of the work of body forces along a flow's current line. A simplified computation, considering the work of forces as a prime mover of the flow (perfect fluid), seems to be 
a good approximation to evaluate velocity evolution versus currents intensity, see equation (11).

$$
\frac{\rho V^{2}}{\frac{I}{S} B h}=c t e
$$

With V: velocity, I: electric current intensity, S: electrodes surface, B magnetic induction, h: height of action of EM forces, $\rho$ fluid density. The latter is well confirmed by the measured normal profile of normal velocity (on the central axis of EM actuator). The profiles are plotted for various currents intensity on Figure 9. The over-plotted solid lines are computed on the base of a similitude governed by equation (11). It demonstrates a very good agreement, which confirms that the EM pumping is mostly balancing the inertia of the flow in the region above the actuator (for $8 \mathrm{~mm}<\mathrm{y}<50 \mathrm{~mm}$ ).

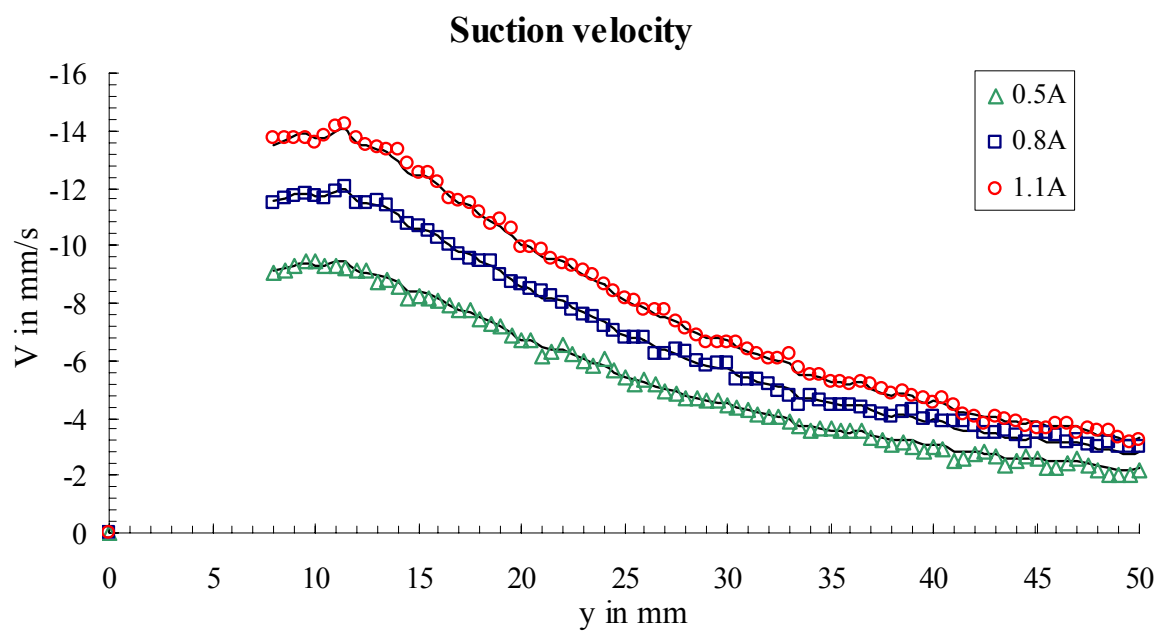

Figure 9: PTV analysis of wall-normal profile of the wall-normal velocity above the actuator axis $(\mathrm{x}=\mathrm{z}=$ 0). $\Delta$ plot corresponds to $\mathrm{I}=0.5 \mathrm{~A}$ i.e. $6600 \mathrm{~A} / \mathrm{m}^{2}$; $\square$ plot corresponds to $\mathrm{I}=0.8 \mathrm{~A}$ i.e. $10500 \mathrm{~A} / \mathrm{m}^{2}$; o plot corresponds to $\mathrm{I}=1.1 \mathrm{~A}$ i.e. $14500 \mathrm{~A} / \mathrm{m}^{2}$. Solid lines over plotted represent similitude prediction (based on forces action) of velocity variation due to electric currents. (Actuator 1999)

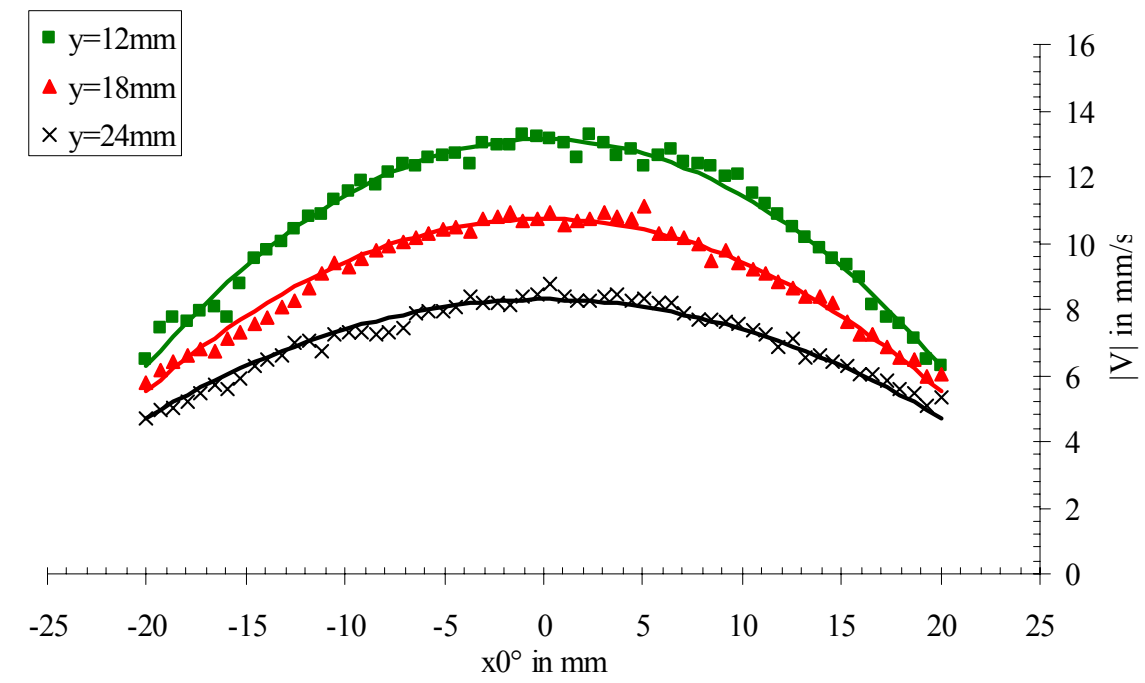

Figure 10: PTV analysis of the transversal profile of the wall normal velocity for different height $y$ $(12 \mathrm{~mm}, 18 \mathrm{~mm}, 24 \mathrm{~mm})$ and a similitude-corrected current of $1 \mathrm{~A} .|\mathrm{V}|$ is in $\mathrm{mm} / \mathrm{s}$, (actuator 1999)

The normal velocity evolution versus $\mathrm{x}$ is given in Figure 10 for various $\mathrm{y}$. This is based on experiments realised for various current: $0.55 \mathrm{~A}, 0.8 \mathrm{~A}$ and $1.1 \mathrm{~A}$, and corrected in similitude to a $1 \mathrm{~A}$ current. This figure complements the Figure 8 and illustrates the 
normal acceleration of the flow near the wall. The interpolation plotted in solid lines on Figure 10 seems to indicate a parabolic transversal profile for suction velocity at a distance from the wall between $12 \mathrm{~mm}<\mathrm{y}<24 \mathrm{~mm}$, see [11].

\subsection{Flow initially at rest: wall jets around the actuator}

Regarding the region very close to the wall, the combination of flow conservation and wall impermeability, drives the EM pumped flow to create wall jets all around the actuator. The image given in Figure 11 represents the superposition of 3 frames taken after a 10 seconds EM actuation in a diagonal and wall-normal plane above the corner of the actuator between magnets and electrodes $\left(\sim 45^{\circ}\right)$.

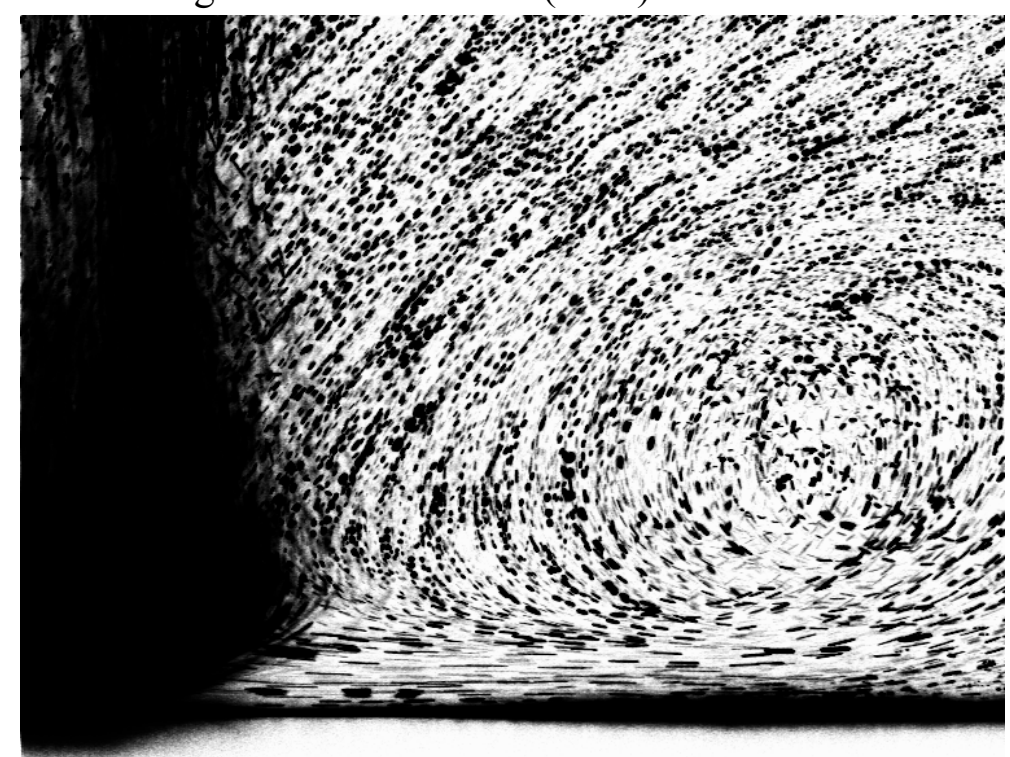

Figure 11: Particles visualization, 3 frames superposition, in a diagonal light sheet $\left(\sim 45^{\circ}\right)$ above the corner of the actuator,. for I=1.1A; 10s EM actuation (flow initially at rest), actuator 1999

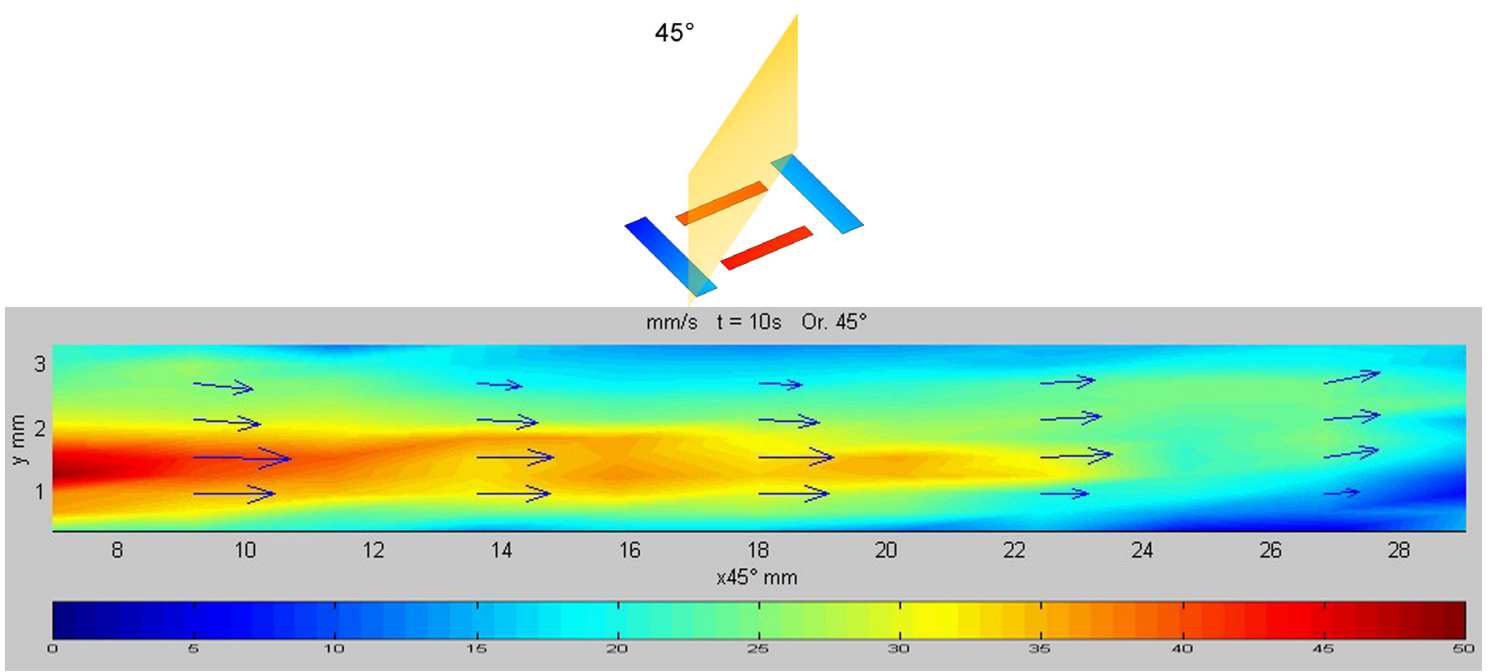

Figure 12: PTV measurement of jet velocity above an EM actuator's corner, $\mathrm{I}=1.1 \mathrm{~A} ; 10 \mathrm{~s}$ actuation. $\mathrm{x} 45^{\circ}=0$ at actuator's corner. (Actuator 1999)

The PTV treatment plotted on Figure 12 gives the velocity in colour-scale for $\mathrm{y}>3 \mathrm{~mm}$ and for $8 \mathrm{~mm}<\mathrm{x}_{45^{\circ}}<28 \mathrm{~mm}$. It clearly demonstrates the existence of corner wall jets. Indeed the velocity here (about $50 \mathrm{~mm} / \mathrm{s}$ ) is much larger than the typical velocity in the suction zone (about $14 \mathrm{~mm} / \mathrm{s}$ see Figure 8 ). In addition the thickness of these corner's jets is very small (about L/10 i.e. $3 \mathrm{~mm}$ ). It appears that these wall jets are related to the development of the coherent structure showed in Figure 5. Wall jets are 
brutally sucked and disappear meanwhile passing under coherent vortical structures. In the present experiments the jet velocity at the actuator's corners is larger than in other region and the thickness of the jet is smaller. These corners region of EM actuator corresponds to region of deficit of EM forces opposed to the local flow.

\subsection{Flow in a seawater tunnel: boundary layers visualization}

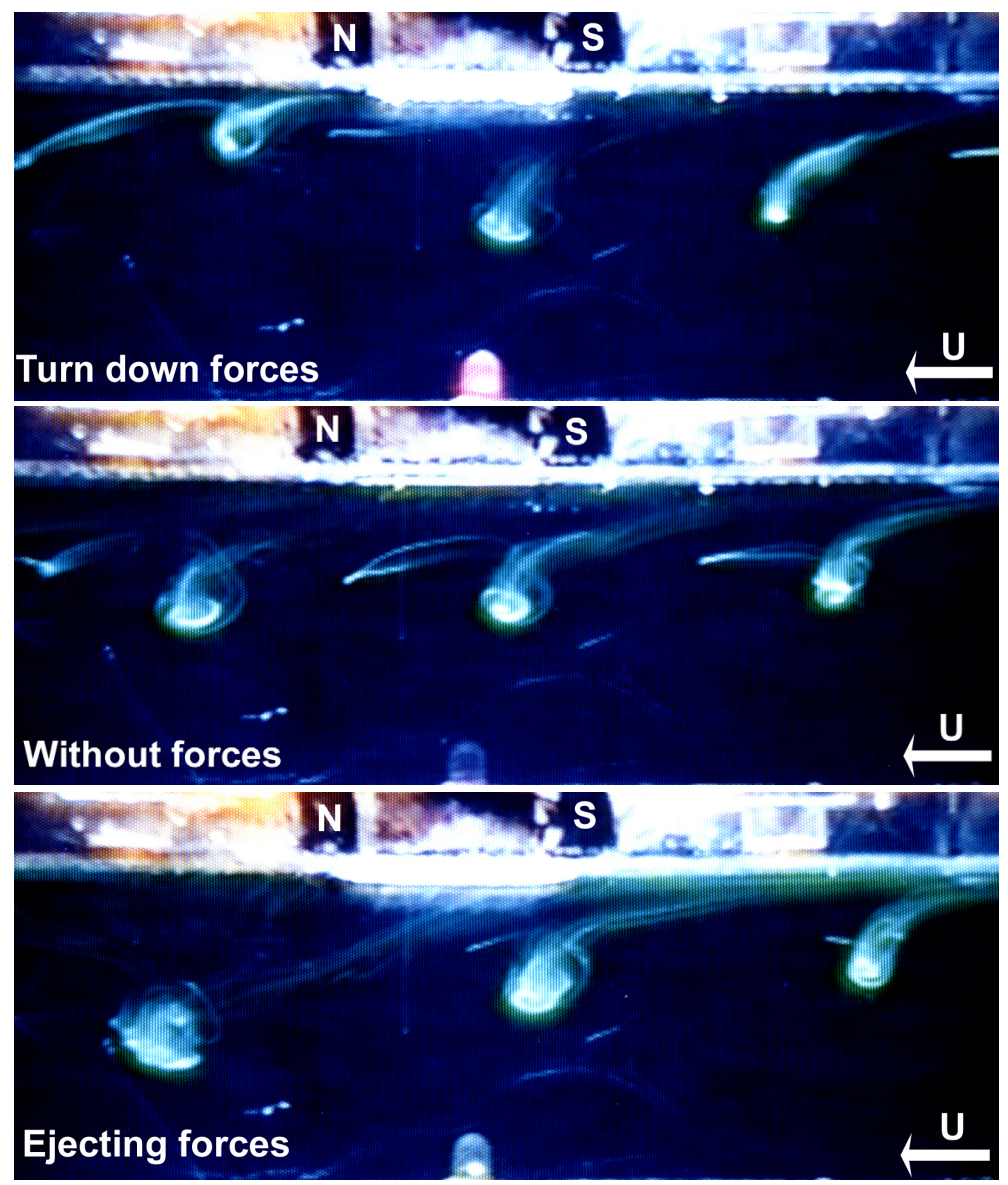

see movie hairpin.mpg

Figure 13: Seawater $(\mathrm{NaCl} \mathrm{35g/1)} \mathrm{tunnel} \mathrm{visualization:} \mathrm{EM} \mathrm{forces} \mathrm{action} \mathrm{on} \mathrm{a} \mathrm{"hairpin} \mathrm{structures} \mathrm{street"}$ generated by a hemisphere protuberance $\left(\mathrm{U}_{\infty} \sim 0.1 \mathrm{~m} / \mathrm{s}, \mathrm{I} \approx 1.1 \mathrm{~A}, \mathrm{~B} \sim 0.65 \mathrm{~T}\right.$ at magnet's pole). Flow is directed from right to left.

The experiments reported here present the effects of EM forces on a boundary layer. Various visualizations are realised in the small seawater tunnel $\left(4 \mathrm{~cm}^{*} 4 \mathrm{~cm}^{*} 1 \mathrm{~m}\right)$. They are presented here to give a qualitative demonstration of the possible use of EM actuators to act directly or around coherent structures similar to the ones observed in a turbulent boundary layer [2]. In order to do so a "synthetic boundary layer" is produced in a wall bounded flow $\left(\mathrm{U}_{\mathrm{ext}} \sim 0.1 \mathrm{~m} / \mathrm{s}\right)$. For this, a "hairpin structure street" is generated by a hemisphere protuberance $[9,12]$. As it is shown on Figure 13 (see also the movie hairpin.mpg) EM forces are able to attract or repulse this synthetic boundary layer, depending on forces sign. It has been observed that, with an attracting effect, structures tend to disappear much faster than without EM action. They degenerate very quickly down-flow the actuator. The competition between the effects of wall-normal flow driven by Lorentz forces (as described in the previous paragraphs) and hairpin structure may be one of the key-parameters controlling the time and capability of "killing" structure by a single shot or multiple (network) shot. 


\section{CONCLUSION}

EM actuator is a novel concept that allows to directly apply in the flow local 3D Lorentz forces. These local body forces are associated with additional forcing terms in Navier Stokes equations as well as in vorticity equation. EM forces, which are mostly present near the wall, are able to pump or to deflect the flow as well as to inject vorticity sources. Consequently each component of velocity or vorticity is altered by EM control either directly during actuation or after it due to a persisting induced velocity (normal component and wall jets) or vorticity.

In regions of the boundary layer where wall-normal velocity is weak, EM actuators impose a novel component of normal velocity different from the one of an ordinary turbulent boundary layer. In regions where turbulent events introduce wall normal velocity, EM control can counteract on and around these events. Finally EM control may be able, on one hand to change the "wall information" of the flow and so to break the turbulence regeneration cycle, on the other hand to alter turbulence by "killing events" as soon as detected.

\section{Acknowledgments}

DGA/BEC and DGA/DSP ; "service repro-visu" of ENSHMG and his director Mr. F. Bonnel; Dr O. Cugat and Dr J. Delamare of LEG-ENSIEG ; Dr. Franck Mc Cluskey ; Dr S. Tardu ; P. Carecchio and M. Kusulga of LEGI.

\section{References}

[1] Nosenchuck D.M., Brown G.L.; 1993, « The direct control of wall shear stress in a turbulent boundary layer », Proc. of the Int. Conf. on Near Wall Turbulent Flows, Elsevier pp 689-698.

[2] Henoch C., Stace J.; 1995, "Experimental Investigation of a Salt Water Turbulent Boundary Layer Modified by an Applied Streamwise Magnetohydrodynamic Body Force", Phys. Fluids 7,(6), pp. 1371-1383.

[3] Weier T., Fey, Gerbeth G., Mutschke G., Avilov; 2000, « Boundary layer control by means of electromagnetic forces » ERCOFTAC bulletin N44, mars 2000 pp37-41.

[4] Robinson S.K.; 1991, « Coherent motions in the turbulent boundary layer», Ann. Rev. Fluid. Mech. $23: 601-39$.

[5] Adrian R.J., Meinhart C.D., Tomkins C.D.; 2000, « Vortex organization in the outer region of the turbulent boundary layer », J. Fluid Mech. Vol 422, pp1-54.

[6] Smith C.R.; 1998, « Vortex developpement and interactions in turbulent boundary layers : implications for surface drag reduction », Proc. of the Int. Symp. on Seawater Drag Reduction, Newport R. I., pp 39-45.

[7] Meng J.C.S.; 1998, « Engineering insight of near wall microturbulence for drag reduction and derivation of a design map for seawater electromagnetic flow control» Proc. of the Int. Symp. on Seawater Drag Reduction, Newport R. I., pp 359-369.

[8] Tardu S.F.; 2001, "Active control of near-wall turbulence by local oscillating blowing" J. Fluid Mech. Vol 439 pp 217-253.

[9] Thibault J-P, Rossi L.; 2000, « Experimental modeling of seawater electromagnetic flow control», ERCOFTAC bulletin $\mathrm{N}^{\circ} 44$, mars 2000 pp 41-49.

[10] Rossi L., Thibault J-P, 2001, "Seawater Flow Control: Wall Normal ElectroMagnetic Actuators", Turbulence Shear Flow Phenomena 2, Stockholm Sweden, vol III, pp 23-28

[11] Rossi L.; 2001, « Contrôle électromagnétique d'écoulement en eau de mer », Thèse de doctorat, Université Joseph Fourier Grenoble, Octobre 2001.

[12] Acarlar M.S., Smith C.R.; 1987, « A study of hairpin vortices in a laminar boundary layer part1 : hairpin vortices generated by a hemisphere protuberance », JFM (1987) vol 175 pp1-41. 